\title{
S05-03
}

\section{COMORBIDITY AND COMORTALITY}

A. Baldacchino', I. Crome ${ }^{2}$

${ }^{1}$ University of Dundee, Dundee, ${ }^{2}$ Academic Psychiatry Unit, Keele University Medical School, Stoke on Trent, UK

Aims: To analyse the nature and extent of data extracted from case files of deceased individuals in contact with health, social care and criminal justice services six months prior to their drug deaths in Scotland during 2003.

Methods: A cross-sectional descriptive analysis of 317 case notes of 237 individuals who had drug related deaths, using a data linkage process, was undertaken. All contacts made with services in the six months prior to death were identified. Information on clinical and social circumstances obtained from case records of social care services, specialist drug treatment, mental health and non-statutory services and the Scottish Prison Service and Criminal Records Office were collated using the Centre for Addiction Research and Education Scotland (CARES) Clinical and Social Circumstances Data Collection Form.

Findings: More than $50 \%(n=237)$ were seen six months prior to their drug death. Sociodemographic details were reported much more frequently than medical problems. While there was information available on ethnicity (49\%), living accommodation (66\%), education and income (52\%), and dependent children (73\%), medical and psychiatric history was recorded in only $12 \%$, blood-borne viral status in $17 \%$, and life events in $26 \%$. This paucity of information was also a feature of the treatment plans and progress recorded for these individuals.

Conclusions: The 237 drug deaths were not a population unknown to services. Highly relevant data about the six-month period prior to death were missing. Improved training to promote in-depth recording, and effective monitoring may result in better understanding and reduction of drug deaths. 